\title{
Compatibility and the Use of Information Processing Strategies
}

\author{
MARCUS SELART, ${ }^{1 *}$ TOMMY GÄRLING, ${ }^{1}$ \\ AND HENRY MONTGOMERY² \\ ${ }^{1}$ Göteborg University, Sweden \\ ${ }^{2}$ Stockholm University, Sweden
}

\begin{abstract}
When a prominent attribute looms larger in one response procedure than in another, a violation of procedure invariance occurs. A hypothesis based on compatibility between the structure of the input information and the required output was tested as an explanation of this phenomenon. It was also compared with other existing hypotheses in the field. The study had two aims: (1) to illustrate the prominence effect in a selection of preference tasks (choice, acceptance decisions, and preference ratings); (2) to demonstrate the processing differences in a matching procedure versus the selected preference tasks. Hence, verbal protocols were collected in both a matching task and in subsequent preference tasks. Silent control conditions were also employed. The structure compatibility hypothesis was confirmed in that a prominence effect obtained in the preference tasks was accompanied by a lower degree of attention to the attribute levels in these tasks. Furthermore, as predicted from the structure compatibility hypothesis, it was found that fewer comparisons between attribute levels were performed in the preference tasks than in the matching task. It was therefore concluded that both these processing differences may explain the occurrence of the prominence effects. (C) 1998 John Wiley \& Sons, Ltd.
\end{abstract}

Journal of Behavioral Decision Making, 11: 59-72, 1998.

KEY WORDS: compatibility; preference elicitation; prominence effect; information structure; verbal protocols

\section{INTRODUCTION}

One of the key principles of normative decision theory is that rational decision makers express their preferences in an invariant way. Thus, the use of different procedures to elicit preferences should not

\footnotetext{
*Correspondence to: Marcus Selart, Göteborg University, Department of Psychology, Haraldsgatan 1, S-413 14 Göteborg, Sweden

Contract grant sponsors: Swedish Council for Research in the Humanities and Social Sciences, Royal Swedish Academy of Sciences
} 
influence the responses (Kahneman and Tversky, 1984). Nevertheless, procedure invariance is often violated by preference reversals, occurring in both risky and riskless situations (e.g. Slovic, Griffin, and Tversky, 1990; Slovic and Lichtenstein, 1983; Tversky, Sattath, and Slovic, 1988; Tversky, Slovic, and Kahneman, 1990; see Payne, Bettman, and Johnson, 1992, and Slovic, 1995, for reviews).

Preference reversals were first demonstrated for gambles (Lichtenstein and Slovic, 1971, 1973; see also Slovic and Lichtenstein, 1983). It was shown that subjects in choosing generally prefer gamble alternatives with large probabilities of winning small amounts. However, in pricing subjects state a higher cash equivalent of gambles with small chances of winning large amounts. The phenomenon has also been demonstrated in riskless multiattribute situations (Slovic et al., 1990; Tversky et al., 1988). For instance, it has been shown that subjects in choice tasks involving two options and two attributes place more weight on the prominent or most important attribute than what they do in a judgmental matching task.

Tversky et al. (1988) argued that this latter phenomenon, which was labelled the prominence effect, occurs because choice and matching tasks evoke different types of decision strategies which place different weight on the prominent attribute. It was suggested that different tasks invoke different kinds of reasoning. Fischer and Hawkins (1993) furthermore proposed that not only matching judgments but judgments in general require quantitative reasoning, whereas choices are characterized by qualitative comparison. The qualitative response in choice was regarded as compatible with a lexicographic decision rule which renders quantitative weighting of attributes unnecessary. In contrast, quantitative judgments were seen as compatible with a quantitative weighting rule. In this way the response mode was considered as priming the focus of attention to the compatible features of the input. This form of compatibility has been referred to as strategy compatibility (Fischer and Hawkins, 1993; Hawkins, 1994; Stone and Schkade, 1991).

Another related mechanism that may produce compatibility effects has been termed scale compatibility (Fischer and Hawkins, 1993; Tversky et al., 1988). Different judgmental response scales have been found to be more or less compatible with the attributes used to describe the items on the input side. For instance, Slovic et al. (1990) found that since a grading scale was more compatible with past grades than with past class ranks, the former attribute was overweighed. In a similar vein, Schkade and Johnson (1989) showed that pricing judgments primed the attention to the winning amount, just like rating scales primed the attention to probabilities. In both cases the attribute most attended to was overweighed. In line with this, Chapman and Johnson (1994) discovered that scale compatibility produces anchoring effects if the anchor and a preference judgment are expressed on the same scale. It was also shown by Chapman and Johnson (1995) that semantic categorization is an important feature of scale compatibility. For instance, in life expectancy evaluations, health items were preferred to commodities, whereas in monetary evaluations commodities were preferred to health items. In an overview of current compatibility theories, Shafir (1995) has recently discussed the scale and strategy compatibility hypotheses.

\section{The structure compatibility framework}

In addition to what should be expected from these accounts of the prominence effect, previous research has shown that a prominent attribute looms larger also in preference ratings as compared to matching. In fact, it has been found that the prominence effect for preference ratings is equally robust and as uniform as the prominence effect in choice (Fischer and Hawkins, 1993; Montgomery et al., 1994; Selart, 1994, 1996; Selart et al., 1994). We therefore adopt another perspective on compatibility in this study. Instead of explaining the prominence effect with compatibility between qualitative or quantitative response modes and decision rule, we propose that the prominence effect is due to differences in compatibility between the structure of the input information and the required output. Structure 
compatibility is assumed to be optimal when the complexity in input and required output is at the same level (the same number of elements and relations between elements). For instance, consider a matching task which includes two treatment alternatives described by two attributes, pain relief and efficiency. The degrees of pain relief and efficiency are expressed on a scale ranging from 0 (no pain relief/efficiency) to 100 (full pain relief/efficiency). For one of the treatments one attribute value is missing. For instance,

$\begin{array}{ccc} & \text { Pain relief } & \text { Efficiency } \\ \text { Treatment A } & 60 & 45 \\ \text { Treatment B } & 45 & ?\end{array}$

The subjects' task is to complete the missing value so that both treatments appear equally attractive. In this task, the required output may be regarded as a difference between two elements which is compared to an input which also consists of a difference between two elements. Irrespective of whether subjects match attribute-wise or alternative-wise, the complexity in input and output is at the same level, implying that structure compatibility is optimal.

However, in choice and preference rating the required output is less complex than the input. Thus, structure compatibility is suboptimal. Consider, for example, a task involving two options and two attributes like in the former case. In choice, the required output then consists of a relationship between two elements (i.e. a preference ordering of two options), whereas the input consists of four elements (the attribute levels for each option) between which there are various relationships. In preference rating the required output is simply one element (e.g. an attractiveness rating on a scale ranging from 1 to 100) and the input consists of two elements (the attribute levels for the judged option). The structure compatibility in choice and preference ratings will therefore be at the same level if it is assumed that the subject bases his or her choice on attractiveness judgments of each option.

The structure compatibility hypothesis thus states that when the structure compatibility is suboptimal, the subject will anchor the required output to a subset of input elements which is at the same complexity level as the required output. As a consequence, these input elements will have a greater weight for the output as compared to the situation when the structure compatibility is optimal. Furthermore, we assume that as a rule the subject will anchor his or her choice or judgment on the more important attribute due to its greater salience in relation to subject's current goals or interests.

This reasoning can be related to the results of Schkade and Kleinmuntz (1994) which suggest that strategies are influenced by the organization of individual items of information into structures. They further suggest that organization strongly influences information acquisition, that is, acquisition is seen as influenced by alternative-wise or attribute-wise stimulus presentation. We think that similar task demands in both choices and preference ratings (sub-optimal structure compatibility) manifest a common organization principle which is different from that used in matching.

\section{Differences between structure compatibility and the competing hypotheses}

It should be noted how our hypothesis differs from the other compatibility hypotheses. Both competing hypotheses (strategy compatibility, scale compatibility) restrict themselves to explain a task effect, that is, the implication of different response procedures. However, structure compatibility may also provide an explanation of other task effects and may also clarify context effects like the effect of similarity between alternatives (see Payne, 1982, and Payne, Bettman, and Johnson, 1993, for a review).

Another difference is that the scale compatibility hypotheses stresses that compatibility will not affect the prominence effect per se but will only enhance the weight of the compatible attribute, whereas a high 
degree of structure compatibility will reduce the prominence effect. On the other hand strategy compatibility implies that only so-called qualitative compatibility (i.e. compatibility between a qualitative response mode and a qualitative decision strategy) will result in a prominence effect.

According to both the competing hypotheses (strategy compatibility, scale compatibility) the prominence effect should not be general among preference tasks but should be limited to choice. The scale compatibility hypothesis (Tversky et al., 1988) predicts an absence of the prominence effect for preference ratings if the scales of the dimensions are made compatible with the output. For instance, in the present study the attribute levels of each pair of alternatives range from 1 to 100 for both the prominent and the non-prominent attribute as does the response scale in the rating task. According to the scale compatibility hypothesis, a more equal weighting of the attributes will hence take place in preference rating than in choice due to this manipulation. The strategy compatibility hypothesis (Fischer and Hawkins, 1993) also predicts a prominence effect in choice, since different kinds of reasoning are assumed to be inherent in choices and judgments independently of the information structure. For instance, a qualitative response mode will trigger a compatible decision strategy which in turn will enhance the prominence effect. As a consequence, both these competing hypotheses predict that there should not exist any processing differences between matching and preference rating which could be related to different degrees of structure compatibility.

\section{Predictions}

The objective of this study is to test the structure compatibility hypothesis. In line with previous findings we predict that prominence effects will occur in both choice and preference ratings in comparison to the matching task. We further test the hypothesis by examining the processing differences between matching, on the one hand, and choice and preference rating, on the other. The prediction is also made that a prominence effect will occur in acceptance decisions in comparison to the matching task, and hence the processing difference between this procedure and the matching task is investigated. The acceptance decisions are introduced in order to study the effects of a qualitative response mode in which the alternatives are evaluated one by one, like in the preference rating task. By introducing acceptance decisions it is hence possible to test the idea that evaluation order is of minor importance for the prominence effect in qualitative procedures (see Shafir, 1993, for a discussion).

According to the structure compatibility hypothesis subjects in the preference tasks will tend to use non-compensatory lexicographic evaluation strategies (i.e. base their response primarily on the prominent attribute), whereas in matching tasks subjects will use compensatory evaluation strategies with a more equal weighting of both the attributes. Of special relevance for the test of the hypothesis is therefore the degree of attention given to the attribute levels in the matching task and in the subsequent preference tasks. This degree is measured by the number of subjects' statements in verbal protocols which are related to alternatives, attributes, or attribute levels.

In the present study, subjects match a number of two-dimensional medical treatments prior to evaluating these treatments using three different response modes. Three predictions are made concerning subjects' information processing. An optimal structure compatibility implies that:

(1) Subjects compare one attribute difference with another in the matching task. They are therefore expected to pay more attention to the attribute levels in this task than they are in the preference tasks. We therefore register how often they attend to the attribute levels.

(2) Subjects are expected to give an equal amount of attention to both attributes in the matching task. In contrast, the use of a lexicographic strategy in the preference tasks is expected to lead to a pronounced level of attention to the prominent attribute. Hence, we register how much attention subjects are giving to the prominent and the non-prominent attribute (Ford et al., 1989). 
(3) Subjects will use the additive-weighting principle in the matching task. They are therefore expected to compare the attribute levels (independently of whether the comparisons are made within or across attributes) in this task more often than they are in the preference tasks. The predictions of the structure compatibility hypothesis and of the other hypotheses are summarized in Exhibit 1.

Exhibit 1 . The different compatibility hypotheses and their predictions

\begin{tabular}{llll}
\hline Hypothesis & Type of effect & Definition of compatibility & Predictions \\
\hline $\begin{array}{l}\text { Strategy } \\
\text { compatibility }\end{array}$ & Task & $\begin{array}{l}\text { Compatibility between a qualitative } \\
\text { response modes and a qualitative } \\
\text { decision strategy or between a } \\
\text { quantitative response mode and a } \\
\text { quantitative decision strategy }\end{array}$ & $\begin{array}{l}\text { Qualitative compatibility will result } \\
\text { in a prominence effect. The effect is } \\
\text { thus restricted to choice procedures. }\end{array}$ \\
& &
\end{tabular}

$\begin{array}{lll}\begin{array}{l}\text { Scale } \\ \text { compatibility }\end{array} & \text { Task } & \begin{array}{l}\text { Compatibility between a judgmental } \\ \text { response scale and an attribute used } \\ \text { to describe the items on the input } \\ \text { side }\end{array} \\ \begin{array}{l}\text { Structure } \\ \text { compatibility }\end{array} & \begin{array}{l}\text { Task and } \\ \text { context }\end{array} & \begin{array}{l}\text { Compatibility between the structure } \\ \text { of the input information and the } \\ \text { required output. }\end{array}\end{array}$

Compatibility will enhance the weight of the compatible attribute. The prominence effect will only occur in choice procedures.

Compatibility will decrease the prominence effect. The prominence effect is not restricted to choice procedures and may also occur in judgments like for instance preference ratings.

Compatibility implies (i) a higher degree of attention to the attribute levels, (ii) an equal attention to both the prominent and the nonprominent attribute, and (iii) the use of a quantitative decision strategy.

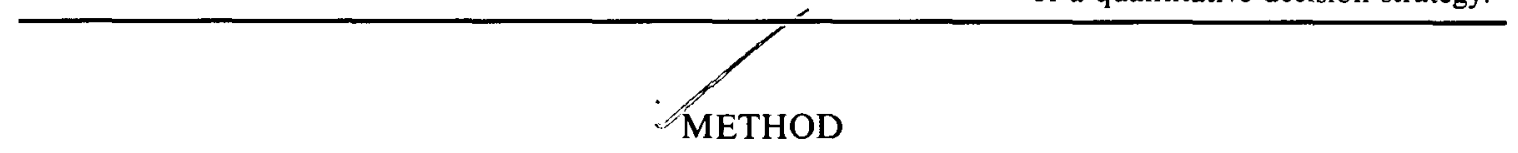

An approach devised by Slovic (1975) is used in which subjects first match two alternatives in order to make them equally attractive. Then they perform a preference task (choice, acceptance decision, or preference rating). In the latter tasks subjects thus evaluate their own matched alternatives. If subjects' preferences at this stage reliably depart from the chance level, for instance in that the prominent attribute is overweighed, then a prominence effect is at hand. It may be questioned how it can be ruled out that the matching procedure in itself will not affect the outcome of the preference tasks. It should therefore be noted that we used the same stimulus material in an earlier study (Selart et al., 1994) using between-group comparisons. A replication of the generality of the prominence effect in the present study would thus indicate a robustness of the effect across the two types of designs.

Verbal protocols are obtained in order to be able to study attention in the different tasks. To be able to test if the verbal protocols are reactive in the matching and the preference tasks (Russo, Johnson, and Stephens, 1989), half of the subjects are not given think-aloud instructions. The reason for using verbal protocols instead of, for instance, information boards is that verbal protocols provide rich naturalistic data which are needed for testing the predictions which are made. Furthermore, there is no indication of that verbal protocols would produce different results in terms of preferences than would information boards (e.g. Ford et al., 1989). 


\section{Subjects}

Seventy-two undergraduate students of psychology at Göteborg University served as subjects in return for financial compensation. Subjects were randomly assigned to six equal-sized groups. In three of these, subjects first performed a matching task followed by a preference task (choice, acceptance decision, or preference rating) while they were thinking aloud. In the other three conditions, the task requirements were the same but subjects were not prompted to think aloud.

\section{Procedure}

Eight problems were each presented twice to subjects in individually randomized orders which were repeated. Each problem consisted of two medical treatments which were described on two attributes. In pretests, one of the attributes had been observed to be more important. The attribute values were expressed on a scale ranging from 1 to 100 . Exhibit 2 displays the attribute levels of the eight problems presented to subjects.

Exhibit 2. Attribute levels of choice problems (medical treatments) presented to subjects

\begin{tabular}{llc}
\hline Attribute & Option 1 & Option 2 \\
\hline 1. Medical care a $(1-100)$ & 65 & 54 \\
Freedom from disturbance $(1-100)$ & 47 & 62 \\
2. Health improvement $(1-100)$ & 61 & 52 \\
Comfort $(1-100)$ & 48 & 64 \\
3. Efficiency $(1-100)$ & 56 & 47 \\
Pain-relief $(1-100)$ & 46 & 58 \\
4. Medical skill $(1-100)$ & 59 & 42 \\
Freedom from fees $(1-100)$ & 35 & 55 \\
5. Food value $(1-100)$ & 66 & 51 \\
Size of food portions $(1-100)$ & 49 & 63 \\
6. Protection against relapse $(1-100)$ & 55 & 40 \\
Program shortage $(1-100)$ & 35 & 52 \\
7. Medical follow-up $(1-100)$ & 56 & 43 \\
Freedom from encroachment (1-100) & 28 & 59 \\
8. Communication with staff $(1-100)$ & 66 & 51 \\
Leisure (1-100) & 46 & 63 \\
\hline
\end{tabular}

aprominent attribute in italics.

On each trial all subjects first performed a matching task. In each pair one attribute value was missing and subjects' task was to supply a value which made the alternatives equally attractive, as exemplified by the following pair (as in the former example efficiency is the prominent and pain-relief the non-prominent attribute):

$\begin{array}{lcc} & \text { Efficiency } & \text { Pain-relief } \\ \text { Treatment 1 } & 56 & 46 \\ \text { Treatment 2 } & 47 & ?\end{array}$

A value was missing for one of the alternatives (either the prominent or non-prominent alternative in a counterbalanced order) the first time the problem was presented. Whether the value was missing on the prominent or non-prominent attribute was counterbalanced for each subject across problems. 
Immediately following the matching task, subjects performed the preference task. For different subjects it either consisted of a preference rating (subjects rated one of the options on a compatible scale ranging from 1 to 100), an acceptance decision of one of the options (subjects indicated whether or not they accepted the option), or a choice between two options. When subjects made preference ratings or acceptance decisions of an alternative in the pair, they were directed to evaluate one alternative the first time and the other alternative the second time in an order which for each subject was counterbalanced across problems.

Half of the subjects were instructed to verbalize their thoughts during the completion of the tasks. If silent for more than approximately 10 seconds, they were reminded of the instructions and prompted to think aloud. The think-aloud reports were tape recorded and later transcribed.

\section{RESULTS}

\section{Matching task}

The analyses of the results rested on the assumption that

$$
u_{\mathrm{P}, \mathrm{p}}+u_{\mathrm{P}, \mathrm{np}}=u_{\mathrm{NP}, \mathrm{p}}+u_{\mathrm{NP}, \mathrm{np}}
$$

where $u_{\mathrm{P}, \mathrm{p}}$ and $u_{\mathrm{P}, \mathrm{np}}$ denote the attractiveness of the levels of the prominent and non-prominent attributes for the prominent option (with the highest value on the prominent attribute) and $u_{\mathrm{NP}, \mathrm{p}}$ and $u_{\mathrm{NP}, \mathrm{np}}$ the corresponding attractiveness of the levels of the prominent and nonprominent attributes for the nonprominent option. The equality in equation (1) thus results from asking subjects to match the options. If the objective attribute levels are denoted $x$ and it is assumed that $u_{i j}=w_{j} x_{i j}$ with $w_{j}$ denoting the attribute weights, then by substitution in equation (1):

$$
w_{\mathrm{p}} / w_{\mathrm{np}}=\left(x_{\mathrm{NP}, \mathrm{np}}-x_{\mathrm{P}, \mathrm{np}}\right) /\left(x_{\mathrm{P}, \mathrm{p}}-x_{\mathrm{NP}, \mathrm{p}}\right)
$$

The data from the matching task were used to verify that one of the attributes was prominent. This was done by computing the ratio between the difference in attribute values for the prominent attribute and the difference in attribute values for the non-prominent attribute. As shown in Exhibit 3, this ratio is reliably higher than 1.0 as was expected $(p<0.05)$. A 2 (think-aloud/silent condition) by 3 (preference task) analysis of variance (ANOVA) yielded no significant main or interaction effects at $p=0.05$.

Exhibit 3. Mean ratios of weights for prominent and non-prominent attributes in the matching task by choice/ rating task condition

\begin{tabular}{lccc}
\hline & Choice & Acceptance decision & Preference rating \\
\hline Think-aloud condition & 1.05 & 1.24 & 1.11 \\
Silent condition & 1.28 & 1.32 & 1.36 \\
\hline
\end{tabular}

\section{The prominence effect}

A measure of the strength of the prominence effect was calculated as follows. A score of 1 was assigned if subjects chose the prominent alternative and a score of 0 if they chose the non-prominent alternative. A score of 1 was similarly assigned if subjects judged the prominent alternative as acceptable and the non-prominent alternative as not acceptable, 0 if the reverse was the case. If both alternatives were judged as acceptable or not acceptable, a score of 0.5 was assigned. If subjects judged their preference to be higher for the prominent alternative, a score of 1 was assigned. If subjects judged their preference to be higher for the non-prominent alternative, a score of 0 was assigned. Ties in preference judgments were given a score of 0.5 . 
Exihibit 4. Mean response scores for choice, acceptance decision, and preference rating

\begin{tabular}{lccc}
\hline & \multicolumn{3}{c}{ Response mode } \\
\cline { 2 - 4 } & Choice & Acceptance decision & Preference rating \\
\hline Think-aloud condition & 0.86 & 0.72 & 0.70 \\
Silent condition & 0.83 & 0.76 & 0.68 \\
\hline
\end{tabular}

In Exhibit 4 the strength of the prominence effect is shown in the six different conditions. In case that: there is not a prominence effect, these numbers would all be 0.50 . Consistent with previous results (Montgomery et al., 1994; Selart et al., 1994), a prominence effect was obtained in each condition. In. a 2 (think aloud/silent condition) by 3 (preference task) ANOVA, no significant main or interaction. effects were revealed at $p=0.05$. Nevertheless, the main effect of type of preference task was close to significant $(p<0.10)$, suggesting that the prominence effect was marginally stronger in choice than. in the other two conditions. The response scores were reliably different from 0.50 in all conditions $(p<0.001)$.

\section{Think-aloud reports}

The processing of the think-aloud reports followed the procedure developed in previous research (e.g. Harte, Westenberg, and van Someren, 1994; Montgomery and Svenson, 1989; Svenson, 1989). Briefly, the coding of the protocols was subdivided into two phases, that is, the division of the protocols into statements (e.g. a sentence with one main idea) and the assignment of categories to each statement. Subsequently, each statement was coded with respect to (1) which of the options, if any, it referred to, (2) which of the attributes, if any, it referred to, and (3) whether subjects compared both options or made an evaluation of a single option. The reliability was determined to be satisfactory for a randomly chosen $10 \%$ of all statements coded by an additional judge. For each subject, attention was calculated by aggregating all statements which referred to the attribute levels. Each statement which referred to a single attribute level received a score of 1 . If a statement reflected a comparison between two attribute levels, each of the compared levels received a score of 1 . All cases of possible comparisons between the four types of attribute levels were coded in this respect. The results are given in Exhibit 5.

As revealed by a 2 (matching task/preference task) by 3 (preference task) by 2 (alternative) by 2 (attribute) by 2 (comparison/single attention) ANOVA with repeated measures on the last three factors, more attribute levels were attended to in the matching task than in the preference tasks, a finding which was in line with the hypothesis, $F(1,33)=16.89, p<0.001$. Furthermore, it was more usual that attribute levels were compared than attended to singly, $F(1,33)=7.89, p<0.01$. This tendency was more pronounced in the matching task than in the preference tasks, $F(1,33)=6.82$, $p<0.01$. The interaction between matching task/preference task and attribute failed however to reach significance, suggesting that the prominent attribute was not reliably more attended to than the nonprominent attribute, $F(1,33)=0.91, p=0.35$. However, a reliable interaction between alternative and attribute showed that there was a difference in attention to the attributes within the prominent alternative in favor of the prominent attribute, $F(1,33)=10.93, p<0.005$. This difference was observed in almost all conditions.

To further substantiate that subjects compare the attribute levels more in the matching task than in the preference tasks, an index was created based on the proportions of the attribute levels compared and the attribute levels which were attended singly. The difference between the frequency of attribute levels compared and attribute levels attended to singly was divided by the total amount of attribute levels compared and attribute levels attended to singly. For all instances in which the total amount 
Exhibit 5. Mean attention to attribute levels in the think-aloud condition

\begin{tabular}{|c|c|c|c|c|}
\hline & \multicolumn{2}{|c|}{ Prominent option } & \multicolumn{2}{|c|}{ Non-prominent option } \\
\hline & $\begin{array}{l}\text { Prominent } \\
\text { attribute }\end{array}$ & $\begin{array}{l}\text { Non-prominent } \\
\text { attribute }\end{array}$ & $\begin{array}{l}\text { Prominent } \\
\text { attribute }\end{array}$ & $\begin{array}{l}\text { Non-prominent } \\
\text { attribute }\end{array}$ \\
\hline \multicolumn{5}{|l|}{ Choice condition } \\
\hline Attribute levels compared & 3.75 & 3.67 & 3.58 & 3.83 \\
\hline $\begin{array}{l}\text { Attribute levels attended singly } \\
\text { Preference task }\end{array}$ & 0.25 & 0.50 & 0.47 & 0.58 \\
\hline Attribute levels compared & 1.00 & 0.58 & 0.50 & 0.08 \\
\hline Attribute levels attended singly & 0.67 & 0.75 & 0.17 & 0.42 \\
\hline \multicolumn{5}{|l|}{$\begin{array}{l}\text { Acceptance decision condition } \\
\text { Matching task }\end{array}$} \\
\hline Attribute levels compared & 2.83 & 2.67 & 2.75 & 2.92 \\
\hline $\begin{array}{l}\text { Attribute levels attended singly } \\
\text { Preference task }\end{array}$ & 2.50 & 1.08 & 1.17 & 2.33 \\
\hline Attribute levels compared & 1.17 & 1.00 & 1.08 & 0.92 \\
\hline Attribute levels attended singly & 0.58 & 0.17 & 0.75 & 0.67 \\
\hline \multicolumn{5}{|l|}{$\begin{array}{l}\text { Preference rating condition } \\
\text { Matching task }\end{array}$} \\
\hline Attribute levels compared & 4.83 & 3.33 & 4.17 & 2.83 \\
\hline $\begin{array}{l}\text { Attribute levels attended singly } \\
\text { Preference task }\end{array}$ & 3.00 & 2.50 & 1.58 & 3.75 \\
\hline Attribute levels compared & 1.00 & 0.75 & 1.67 & 1.42 \\
\hline Attribute levels attended singly & 1.58 & 0.83 & 1.17 & 1.00 \\
\hline
\end{tabular}

equalled 0 , mean values for each term were used as replacements, in order to save cases in the analysis (division with 0 would delete cases). This recoding procedure included about $35 \%$ of all data. The missing data hence consisted of statements made by subjects in which no explicit reference was made to the attribute levels. It did therefore not indicate silence. The means of the proportions are presented in Exhibit 6 . As was shown by a 2 (matching task/preference task) by 3 (preference task) by 2 (alternative)

Exhibit 6. Mean index of attention to attibute levels in the think-aloud condition

\begin{tabular}{lccccc}
\hline & \multicolumn{2}{c}{ Prominent option } & & \multicolumn{2}{c}{ Non-prominent option } \\
\cline { 2 - 3 } \cline { 5 - 6 } & $\begin{array}{c}\text { Prominent } \\
\text { attribute }\end{array}$ & $\begin{array}{c}\text { Non-prominent } \\
\text { attribute }\end{array}$ & & $\begin{array}{c}\text { Prominent } \\
\text { attribute }\end{array}$ & $\begin{array}{c}\text { Non-prominent } \\
\text { attribute }\end{array}$ \\
\hline $\begin{array}{l}\text { Choice condition } \\
\text { Matching task }\end{array}$ & $0.76^{\mathrm{a}}$ & 0.65 & & 0.59 & 0.56 \\
Preference task & 0.37 & 0.11 & & 0.41 & -0.65 \\
$\begin{array}{l}\text { Acceptance decision condition } \\
\text { Matching task }\end{array}$ & 0.15 & 0.41 & & 0.45 & 0.11 \\
$\begin{array}{l}\text { Preference task } \\
\text { Preference rating condition }\end{array}$ & 0.31 & 0.66 & & 0.19 & 0.12 \\
$\begin{array}{l}\text { Matching task } \\
\text { Preference task }\end{array}$ & 0.26 & 0.14 & & 0.40 & -0.21 \\
\hline
\end{tabular}

${ }^{a}$ Positive numbers indicate that subjects predominantly compared the attribute levels, negative numbers that they predominantly attended to the attribute levels singly. 
by 2 (attribute) ANOVA, the proportions of compared attribute levels were higher in the matching task than in the preference tasks, $F(1,33)=10.97, p<0.01$. A reliable interaction with preference task revealed that this tendency was most profound in the choice condition, $F(2,33)=6.44, p<0.01$. Furthermore, it was found that the proportion of compared attribute levels was higher for the prominent than for the non-prominent attribute, $F(1,33)=8.68, p<0.01$. This effect was once again strongest in the choice condition, $F(2,33)=5.24, p<0.05$. In addition, it was found that the proportion of compared attribute levels was higher for the prominent option in choice, but lower for the same option in preference rating, $F(2,33)=6.63, p<0.01$. It was also observed that the proportion of compared attribute levels was higher for the prominent attribute of the non-prominent option than for the prominent attribute of the prominent option, $F(1,33)=60.55, p<0.001$. This tendency was most evident in the preference rating condition.

\section{DISCUSSION}

A method devised by Slovic (1975) was used in which subjects matched two alternatives in order to make them equally attractive prior to performing a preference task (choice, acceptance decision, or: preference rating). Verbal protocols were collected in the experimental condition, but not in the control condition. A prominence effect was obtained for choices. This finding replicated the results of Tversky et al. (1988) who demonstrated the effect using a between-subjects design entailing choice and matching. In addition, prominence effects for acceptance decisions and preference ratings were also observed in the present study. We used a silent control condition in order to test the confounding effects of giving verbal protocols (Russo, Johnson, and Stephens, 1989). The response scores in this condition did not depart from those in the think-aloud condition, indicating that a prominence effect: was obtained in both conditions. Consequently, it was suggested that the verbal protocols did not cause any reactivity in this respect.

It was hypothesized that the prominence effect may be explained by assuming that the preference tasks are characterized by a suboptimal structure compatibility. In these tasks a complete set of attribute levels were presented in contrast to the initial matching task. However, the preference tasks were heterogeneous in the sense that two of them were qualitative (choice, acceptance decision) whereas one was quantitative in nature (preference rating). Despite this, no reliable differences in the strength of the prominence effect were obtained.

The results obtained cannot be explained by strategy or scale compatibility. In strategy compatibility the distinction between qualitative and quantitative response modes is highlighted (Fischer and Hawkins, 1993; Tversky et al., 1988). This principle explains why the most important attribute looms larger in choice than in matching, with reference to the qualitative/quantitative distinction. Apparently, the prominence effect obtained for preference ratings cannot be explained by this principle. Scale compatibility implies that the input is weighted more heavily if it is expressed on the same scale as the response (Tversky et al., 1988). Our attribute levels ranged from 1 to 100 for both the prominent and the non-prominent attribute, as did our response scale for preference ratings. According to the scale compatibility principle, this should have resulted in a more equal weighting of the attributes in preference rating than in the choice and acceptance tasks.

The verbal protocols showed that the prominence effect was accompanied by a lower degree of attention to the attribute levels in the preference tasks than in the matching task. Subjects also less frequently compared attribute levels in the preference tasks than in the matching task. The results were thus in line with the predictions from the structure compatibility hypothesis. However, the enhanced attention to the prominent attribute that should have been found in the preference tasks, reflecting a lexicographic decision strategy, was not observed. 
An important issue is therefore what can be concluded about subjects' use of decision strategies from the processing data. Our prediction was that a compensatory strategy (the additive weighting rule) should be used in the matching task, while a non-compensatory strategy (the lexicographic rule) should be used in the preference tasks. To be able to perform the matching task, subjects must make trade-offs along dimensions, and thus it seems reasonable to assume that a compensatory strategy had to be used. In line with this, our data revealed that the information processing of the matching task was based on more information and included more comparisons between the attribute levels. These are typical signs of compensatory strategies.

The generality of the prominence effect among the preference tasks indicated that a lexicographic strategy was used in these tasks. Hence, subjects rank ordered the dimensions and systematically preferred the option with the highest value on the most important attribute. However, as revealed by the processing data, the use of this strategy did not imply that more attention was given to the prominent attribute in comparison to the non-prominent attribute. Nevertheless, the obtained prominence effect across the preference tasks and the observed lower degrees of attention to the attribute levels in these response modes, confirm the structure-compatibility hypothesis (Montgomery et al., 1994; Selart et al., 1994; Selart, 1996). The observed process differences also provide an account of how the principle functions at a cognitive level.

Although there were no differences to be observed between the mean weight ratios of the matching task among conditions, minor differences could be observed on the process level of the matching task among conditions in terms of the attention given to the attribute levels (see Exhibits 5 and 6). This indicates that subjects in the matching task are more sensitive to different conditions in their processing of the information than in their elicitation of the weights.

There have been several studies dealing with partly the same issues as have been raised in the present study. Recently, such a study was conducted by Hawkins (1994). He introduced a set of hypotheses of which a majority concerned processing differences (response time, fixation time) between choice and matching. A computerized information board technique was used in the empirical investigation. First, the prominence effect was replicated. It was also revealed that the matching task in relation to choice had (1) longer total response times, (2) more acquisitions, and (3) longer relative fixations time for the prominent attribute. Furthermore, it was found that a prominence effect between choice and matching could be attributed to relative attention to the prominent attribute in choice. These results show clear similarities with those obtained in our verbal protocol analysis which also focused on the choicematching discrepancy. However, in addition to these results our model accounts for similar processing differences observed between matching and preference rating. By technical reasons, we have nevertheless only been able to confirm longer total response times and more acquisitions in the matching task, and have not been able to corroborate longer relative fixations times spent on the prominent attribute.

Our results may also be related to those obtained by Schkade and Kleinmuntz (1994). They emphasized the role of information structure on preference elicitation. It was revealed that the acquisition of information is influenced by how the stimulus is presented. For instance, it was shown to be crucial whether the attribute levels were presented attribute-wise or alternative-wise. The importance of information structure was also noted by Payne et al. (1992). As in the present study, they investigated the contingency of preferences on task demands. Subjects were asked to make comparisons between input data, before they were instructed to elicit their preferences in a subsequent task. Examples of such comparisons were information transformations (e.g. rounding off, standardizing, or performing calculations), rearranging information (e.g. changing the order of the options or attributes), or eliminating information (see also Russo, 1977; Ranyard, 1989). It was found that subjects engaged in these comparison tasks prior to the preference elicitation task were to a higher extent using alternativebased strategies when processing the restructured material. 
Another related account was presented by Payne (1982). He suggested that two general classes of variables play a major part for how subjects construct their preferences. These are the task and the context effects. Task effects can be related to manipulations of the general structure of the decision, including response mode, number of options or attributes, time pressure, and presentation constraints. Context effects, on the other hand, can be connected to manipulations of the content of the decision problem, involving attribute values, similarity of alternatives, attribute covariation, and overall attractiveness of alternatives. It seems important to clarify that although structure compatibility effects in this study are due to manipulations of the task, this type of compatibility has also been demonstrated to be inherent in context effects. For instance, it has been found that the manipulation of different value ranges between the attribute levels influence the magnitude of the prominence effect (Selart, 1996).

In summary, our results suggest a contingent weighting mechanism. As indicated by the generality of the prominence effect, supported by a lower degree of attention to the attribute levels, especially in terms of performed comparisons, these results provide a clear support for a structure compatibility explanation. The hypothesis is also supported by a higher proportion of compared attribute levels of the prominent attribute than of the non-prominent attribute. Taking previous research into account, it must be pointed out that features like scale type, semantic characteristics, and information structure may all produce overweighing phenomena, like the prominence effect. It might be argued that to be able to study the effect of information presentation in a broader sense, different ways of presenting the information should be compared while using the same evaluation task. Despite the fact that such an analysis was not performed in this study, it seems clear that the notion of structure compatibility provides an account at a more specific level of how the construction of preferences may depend on information presentation. It is also important to note that other variables like positive versus negative comparisons between the attribute levels might also have explanatory value for the prominence effect.

\section{AUTHORS' NOTE}

The study was financially supported by grants to the first author from the Swedish Council for Research in the Humanities and Social Sciences and from the Royal Swedish Academy of Sciences. A previous version of the paper was presented at the 7th conference of the European Society for Cognitive Psychology (ESCOP), Lisbon, Portugal, in September 1994. We thank Härma Savioja-Jensen for assistance in the documentation and analysis of the verbal protocols.

\section{REFERENCES}

Chapman, G. B. and Johnson, E. J. 'The limits of anchoring', Journal of Behavioral Decision Making, 7 (1994), 223-42.

Chapman, G. B. and Johnson, E. J. 'Preference reversals in monetary and life expectancy evaluations', Organizational Behavior and Human Decision Processes, 62 (1995), 300-17.

Fischer, G. W. and Hawkins, S. A. 'Strategy compatibility, scale compatibility, and the prominence effect', Journal of Experimental Psychology: Human Perception and Performance, 19 (1993), 580-97.

Ford, J. K., Schmitt, N., Schechtman, S. L., Hults, B. M. and Doherty, M. L. 'Process tracing methods: Contributions, problems, and neglected research issues', Organizational Behavior and Human Decision Processes, 43 (1989), 75-117.

Harte, J. M., Westenberg, M. R. M. and van Someren, M. 'Process models of decision making', Acta Psycho-. logica, 87 (1994), 95-120.

Hawkins, S. A. 'Information processing strategies in riskless preference reversals: The prominence effect', Organizational Behavior and Human Decision Processes, 59 (1994), 1-26.

Kahneman, D. and Tversky, A. 'Choices, values, and frames', The American Psychologist, 39 (1984), $341-50$. 
Lichtenstein, S. and Slovic, P. 'Reversals of preference between bids and choices in gambling decisions', Journal of Experimental Psychology, 89 (1971), 46-55.

Lichtenstein, S. and Slovic, P. 'Response-induced reversals of preference in gambling: An extended replication in Las Vegas', Journal of Experimental Psychology, 101 (1973), 16-20.

Montgomery, H., Selart, M., Gärling, T. and Lindberg, E. 'The judgment-choice discrepancy: Noncompatibility or restructuring?' Journal of Behavioral Decision Making, 7 (1994), 145-55.

Montgomery, H. and Svenson, O. 'A think-aloud study of dominance structuring in decision processes', In Montgomery, H. and Svenson, O. (eds), Process and Structure in Human Decision Making, Chichester: Wiley, 1989.

Payne, J. W. 'Contingent decision behavior', Psychological Bulletin, 92 (1982), 382-402.

Payne, J. W., Bettman, J. R., Coupey, E. and Johnson, E. J. 'A constructive process view of decision making: Multiple strategies in judgment and choice', Acta Psychologica, 80 (1992), 107-41.

Payne, J. W., Bettman, J. R. and Johnson, E. J. 'Behavioral decision research: A constructive processing perspective', Annual Review of Psychology, 43 (1992), 87-131.

Ranyard, R. 'Structuring and evaluating simple monetary risks', in Montgomery, H. and Svenson, O. (eds), Process and Structure in Human Decision Making, Chichester: Wiley, 1989.

Russo, J. E. 'The value of unit price information', Journal of Marketing Research, 14 (1977), 193-201.

Russo, J. E., Johnson, E. J. and Stephens, D. L. 'The validity of verbal protocols', Memory \& Cognition, 17 (1989), 759-69.

Schkade, D. A. and Johnson, E. J. 'Cognitive processes in preference reversals', Organizational Behavior and Human Decision Processes, 44 (1989), 203-31.

Schkade, D. A. and Kleinmuntz, D. N. 'Information displays and choice processes: Differential effects of organization, form and sequence', Organizational Behavior and Human Decision Processes, 57 (1994), 319-37.

Selart, M. Preference Reversals in Judgment and Choice: The Prominence Effect, Doctoral dissertation, Göteborg University, Sweden, 1994.

Selart, M. 'Structure compatibility and restructuring in judgment and choice', Organizational Behavior and Human Decision Processes, 65 (1996), 106-16.

Selart, M., Montgomery, H., Romanus, J. and Gärling, T. 'Violations of procedure invariance in preference measurement: Cognitive explanation', European Joural of Cognitive Psychology, 6 (1994), 417-36.

Shafir, E. 'Choosing versus rejecting: Why some options are both better and worse than others', Memory \& Cognition, 21 (1993), 546-56.

Shafir, E. 'Compatibility in cognition and decision', in Busemeyer, J., Hastie, R. and Medin, D. L. (eds), Decision Making from a Cognitive Perspective. The Psychology of Learning and Motivation, Vol.32 (pp. 247-74), San Diego: Academic Press, 1995.

Slovic, P. 'Choice between equally valued alternatives', Journal of Experimental Psychology: Human Perception and Performance, 1 (1975), 280-87.

Slovic, P. 'The construction of preference', American Psychologist, 50 (1995), 364-71.

Slovic, P., Griffin, D. and Tversky, A. 'Compatibility effects in judgment and choice', in Hogarth, R. M. (ed.), Insights in Decision Making: A Tribute to Hillel J. Einhorn (pp. 5-27), Chicago, IL: University of Chicago Press, 1990.

Slovic, P. and Lichtenstein, S. 'Preference reversals: A broader perspective', American Economic Review, 73 (1983), 623-38.

Stone, D. N. and Schkade, D. A. 'Numeric and linguistic information representation in multiattribute choice', Organizational Behavior and Human Decision Processes, 49 (1991), 42-59.

Svenson, $O$. 'Eliciting and analysing verbal protocols in process studies of judgment and decision making', Montgomery, H. and Svenson, O. (eds), Process and Structure in Human Decision Making. Chichester: Wiley, 1989.

Tversky, A., Sattath, S. and Slovic, P. 'Contingent weighting in judgment and choice', Psychological Review, 95 (1988), 371-84.

Tversky, A., Slovic, P. and Kahneman, D. 'The causes for preference reversals', The American Economic Review, 80 (1990), 204-17. 
Authors' biographies:

Marcus Selart is Assistant Professor of Psychology at Göteborg University. His research mainly concern various issues in behavioral decision making.

Tommy Gärling is Professor of Psychology at Göteborg University. His main research areas are behavioral decision making and environmental psychology.

Henry Montgomery is Professor of Cognitive Psychology at Stockholm University. His current research deals with perspective shifts in evaluations of decision outcomes.

Authors' addresses:

Marcus Selart and Tommy Gärling, Department of Psychology, Göteborg University, Haraldsgatan 1, S-413 14 Göteborg, Sweden.

Henry Montgomery, Department of Psychology, Stockholm University, S-106 91 Stockholm, Sweden. 
Copyright of Journal of Behavioral Decision Making is the property of John Wiley \& Sons, Inc. / Business and its content may not be copied or emailed to multiple sites or posted to a listserv without the copyright holder's express written permission. However, users may print, download, or email articles for individual use. 